I. Math. Soc. Japan

Vol. 13, No. 4, 1961

\title{
On ordinal diagrams
}

\author{
By Akiko KINo
}

(Received Jan. 23, 1961)

G. Takeuti developed the theory of ordinal diagrams of order $n$ (where $n$ is a positive integer) in [2], and generalized it to the theory of ordinal diagrams constructed from well-ordered sets $I, A$, and $S$ in [3]. It was necessary to consider $S$ in order to prove the accessibility for $\operatorname{Od}(I, A, S)$ (the system of ordinal diagrams constructed from $I, A$ and $S$ ) given in [3]. But $S$ did not serve to extend the system of ordinal diagrams. In fact, if we denote $\mathrm{Od}(I, A, S)$ and $\mathrm{O}(I, A, S)$ with empty $S$ by $\mathrm{Od}(I, A)$ and $\mathrm{O}(I, A)$ respectively, we can embed $\operatorname{Od}(I, A, S)($ or $\mathrm{O}(I, A, S))$ into $\mathrm{Od}(\{*\} \cup I, A \cup S)$ (or $\mathrm{O}(\{*\} \cup I, A \cup S)$ ), where $*$ is distinct form any element of $I, A$ and $S$; the notation $A \cup S$ means the well-ordered set obtained from $A$ and $S$ by keeping the orders in themselves and setting the elements of $A$ before the elements of $S$. The embedding is defined as follows:

1. If $\alpha \in A$, then $\alpha^{*}$ is $\alpha$.

2. If $\alpha$ is of the form $\left(\alpha_{0}, s\right)$, then $\alpha^{*}$ is $\left(*, \alpha_{0}^{*}, s\right)$.

3. If $\alpha$ is of the form $\left(i, \alpha_{1}, \alpha_{2}\right)$, then $\alpha^{*}$ is $\left(i, \alpha_{1}^{*}, \alpha_{2}^{*}\right)$.

4. If $\alpha$ is of the form $\alpha_{1} \# \alpha_{2}$, then $\alpha^{*}$ is $\alpha_{1}^{*} \# \alpha_{2}^{*}$.

Now we can simplify the proof of the accessibility of $\operatorname{Od}(I, A, S)$ in a similar way as in $\S 2$ of [2], whether $S$ is empty or not (cf. $\S 2$ of this paper). In this paper, we shall construct a system $\operatorname{Od}(I)$, namely " the system of ordinal diagrams constructed from a well-ordered set $I$ " (in $\S 1$ ), and prove that the system is well-ordered for the given orderings in a similar way as in [2] (in $\S 2)$. Then we shall show that the present system is a generalization of previous systems. In fact, $\operatorname{Od}(I, A)$ is embedded into $\operatorname{Od}(I \cup A)$ in $\S 3$. By the way, we shall show that a formal theory of $\operatorname{Od}(I, A)$ can be formalized in the system developed in [5] and is consistent.

The author wishes to express her heart-felt thanks to Prof. G. Takeuti for his valuable advice and kind encouragement in the preparation of this paper.

\section{$\S 1$. Ordinal diagrams constructed from $I$.}

Let $I$ be a well-ordered set with the order $<^{*}$ and $o$ be the first element of $I$. In this section, we shall construct a kind of system of ordinal diagrams, called ordinal diagrams constructed from $I$ and denoted by $\operatorname{Od}(I)$. Though 
the word o.d. is used in [2] and in [3] to denote an element of ordinal diagrams developed there, we use it instead of 'an element of $\operatorname{Od}(I)$ ' for simplification throughout this and the next sections.

1. $\operatorname{Od}(I)$ is defined recursively as follows:

1.1. If $i \in I$, then $i$ is an o. d.

1.2. If $\alpha$ and $\beta$ are o. d.'s, then $(\alpha, \beta)$ is an o.d.

1.3. If $\alpha$ and $\beta$ are o.d.'s, then $\alpha \# \beta$ is an o.d.

2. An o. d. $\alpha$ is called a $c . o . d$. (connected ordinal diagram constructed from $I$ ), if and only if the operation used in the final step of construction of $\alpha$ is not \#.

3. Let $\alpha$ be an o. d. We define components of $\alpha$ recursively as follows:

3.1. If $\alpha$ is a c. o. d., then $\alpha$ has exactly one component which is $\alpha$ itself.

3.2. If $\alpha$ is an o. d. of the form $\alpha_{1} \# \alpha_{2}$, then the components of $\alpha$ are the components of $\alpha_{1}$ and of $\alpha_{2}$.

4. Let $\alpha$ and $\beta$ be o. d.'s. We define $\alpha=\beta$ recursively as follows :

4.1. Let $\alpha \in I$. Then $\alpha=\beta$, if $\beta$ is an element of $I$ and equal to $\alpha$ in $I$.

4.2. Let $\alpha$ be of the form $\left(\alpha_{0}, \alpha_{1}\right)$. Then $\alpha=\beta$ if $\beta$ is of the form $\left(\beta_{0}, \beta_{1}\right)$ and $\alpha_{0}=\beta_{0}$ and $\alpha_{1}=\beta_{1}$.

4.3. Let $\alpha$ have $k$ components $\alpha_{1}, \cdots, \alpha_{k}(k>1)$. Then $\alpha=\beta$, if $\beta$ has $k$ components, and $\beta_{1}, \cdots, \beta_{k}$ being these components, there exists a permutation $\left(m_{1}, \cdots, m_{k}\right)$ of $(1, \cdots, k)$ such that $\alpha_{n}=\beta_{m_{n}}$ for $n=1, \cdots, k$.

4.4. $\beta=\alpha$ if $\alpha=\beta$.

5. Let $\alpha$ be an o.d. The rank of $\alpha$ means the sum of the number of $($, and $\#$ in $\alpha$.

6. Let $\alpha, \beta$ and $\xi$ be o. d.'s. We define the relations $\beta \subset_{\xi} \alpha$ (to read: $\beta$ is a $\xi$-section of $\alpha$ ) and $\beta<_{\xi} \alpha, \beta<_{\infty} \alpha$ and 'index of $\alpha$ ' simultaneously as follows :

6.1. If $\alpha, \beta \in I$, then $\beta<_{\xi} \alpha$ and $\beta<_{\infty} \alpha$ means $\beta<^{*} \alpha$.

6.2. Let one (or both) of $\alpha$ and $\beta$ be not a c.o.d., and the components of $\alpha$ and $\beta$ be $\alpha_{1}, \cdots, \alpha_{h}$ and $\beta_{1}, \cdots, \beta_{k}$ respectively. $\beta<_{\xi} \alpha$ holds if one of the following conditions is satisfied:

6.2.1. There exists an $\alpha_{m}(1 \leqq m \leqq h)$ such that $\beta_{n}<_{\xi} \alpha_{m}$ holds for every $n(1 \leqq n \leqq k)$.

6.2.2. $h>1, k=1$ and $\beta_{1}=\alpha_{m}$ for some $m(1 \leqq m \leqq h)$.

6.2.3. $h>1, k>1$ and there exist an $\alpha_{m}(1 \leqq m \leqq h)$ and a $\beta_{n}(1 \leqq n \leqq k)$ such that $\alpha_{m}=\beta_{n}$ and

$$
\beta_{1} \# \cdots \# \beta_{n-1} \# \beta_{n+1} \# \cdots \# \beta_{k}<_{\xi} \alpha_{1} \# \cdots \# \alpha_{m-1} \# \alpha_{m+1} \# \cdots \# \alpha_{h} \text {. }
$$

$\beta<_{\infty} \alpha$ holds if one of 6.2.1-6.2.3 with $\infty$ in place of $\xi$ is fulfilled. 
6.3. If $\alpha \in I$, then $\beta \subset_{\xi} \alpha$ never holds.

6.4. Let $\alpha$ be of the form $\left(\alpha_{0}, \alpha_{1}\right)$.

6.4.1. If $\xi<_{0} \alpha_{0}$, then $\beta \subset_{\xi} \alpha$ if and only if $\beta \subset_{\xi} \alpha_{1}$.

6.4.2. If $\xi=\alpha_{0}$, then $\beta \subset_{\xi} \alpha$ if and only if $\beta$ is $\alpha_{1}$.

6.4.3. If $\alpha_{0}<_{o} \xi$, then $\beta \subset_{\xi} \alpha$ never holds.

6.5. Let $\alpha$ be of the form $\alpha_{1} \# \alpha_{2}$. Then $\beta \subset_{\xi} \alpha$ if and only if either $\beta \subset_{\xi} \alpha_{1}$ or $\beta \subset_{\xi} \alpha_{2}$ holds.

6.6. $\xi$ is called an index of $\alpha$, if $\alpha$ has a $\xi$-section.

In the following we shall simply say ' $\xi$ is less (or greater) than $\eta$ ' and ' $\xi$ is the minimum (or maximum)' in place of ' $\xi$ is less (or greater) than $\eta$ in the sense of $<_{0}$ ' and ' $\xi$ is the minimum (or maximum) in the sense of $<_{0}$, respectively.

6.7. Let $\alpha$ and $\beta$ be c. o. d's. If there exists an index $\eta$ of $\alpha$ and/or $\beta$ such that $\xi<_{o} \eta$, then $\xi^{+}$is defined to be the minimum of such indices; otherwise, $\xi^{+}$is defined to be $\infty$. Then $\beta<_{\xi} \alpha$, if and only if one of the following conditions is fulfilled:

6.7.1. There exists a $\xi$-section $\alpha_{0}$ of $\alpha$ such that $\beta \leqq \xi \alpha_{0}$.

6.7.2. $\beta_{0}<_{\xi} \alpha$ for every $\xi$-section $\beta_{0}$ of $\beta$ and $\beta<\xi^{+} \alpha$.

6.8. Let $\alpha$ and $\beta$ be c. o. d,'s of the form $\left(\alpha_{0}, \alpha_{1}\right)$ and $\left(\beta_{0}, \beta_{1}\right)$ respectively. $\beta<_{\infty} \alpha$ if and only if one of the following conditions is fulfilled:

6.8.1. $\beta_{0}<_{0} \alpha_{0}$.

6.8.2. $\beta_{0}=\alpha_{0}$ and $\beta_{1}<_{\alpha_{0}} \alpha_{1}$.

6.9. Let $\alpha \in I$ and $\beta$ be a c.o.d. of the form $\left(\beta_{0}, \beta_{1}\right) . \alpha<_{\infty} \beta$ if $\alpha \leqq_{0} \beta_{0}$, $\beta<_{\infty} \alpha$ if $\beta_{0}<_{0} \alpha$.

Under these definitions the following propositions are easily proved.

Proposition $1 .=$ is an equivalence relation between o.d.'s.

Proposition 2. Let $\alpha_{1}, \alpha_{2}, \beta_{1}, \beta_{2}$ be o.d.'s. $\alpha_{1}=\beta_{1}$ and $\alpha_{2}=\beta_{2}$ imply $\alpha_{1} \# \alpha_{2}=\beta_{1} \# \beta_{2}$.

Proposition 3. Let $\alpha_{1}, \alpha_{2}, \beta_{1}, \beta_{2}$ be o.d.'s and $\gamma$ be an o.d. or $\infty$. Then $\alpha_{1}=\beta_{1}, \alpha_{2}=\beta_{2}$ and $\alpha_{1}<_{\gamma} \alpha_{2}$ imply $\beta_{1}<_{\gamma} \beta_{2}$.

Proposition 4. Each of the relations $<_{\xi}$, where $\xi$ is an o.d. or $\infty$, defines a linear order between o. d.'s.

Proposition 5. Let $\alpha$ and $\beta$ be o.d.'s. Then $\beta<_{\xi}(\alpha, \beta)$ for every $\gamma$ such that $\gamma \leqq_{0} \alpha$.

\section{$\S 2$. Accessibility of $\operatorname{Od}(I)$.}

Let $S$ be a system with a linear order $<$. An element $s$ of $S$ is called 'accessible in $S$ (or accessible for $<$ )', if the subsystem of $S$ consisting of elements, which are not greater than $s$ in the sense of $<$, is well-ordered. $S$ is called accessible, if the whole system is well-ordered by $<$. 
1. Let $\alpha$ and $\beta$ be o. d.'s. We define a relation $\beta \leqslant \alpha$ (to read; $\beta$ is a value of $\alpha$ ) as follows :

1.1. If $\alpha \in I$, then $\alpha$ has no value, that is, $\beta \leqslant \alpha$ never holds.

1.2. Let $\alpha$ be not a c. o. d. and have components $\alpha_{1}, \cdots, \alpha_{k}$. Then $\beta \leqslant \alpha$, if $\beta \leqslant \alpha_{m}$ for some $m(1 \leqq m \leqq k)$.

1.3. Let $\alpha$ be of the form $\left(\alpha_{0}, \alpha_{1}\right)$. Then $\beta \leqslant \alpha$, if $\beta$ is $\alpha_{0}$ or $\beta \leqslant \alpha_{0}$ or $\beta \Leftarrow \alpha_{1}$.

2. Let $\alpha$ and $\beta$ be o.d.'s. $\beta$ is called a $\left(\xi_{1}, \cdots, \xi_{n}\right)$-section of $\alpha$, if the following conditions are fulfilled:

2.1. $\xi_{1} \leqq_{o} \xi_{2} \leqq_{0} \cdots \leqq_{o} \xi_{n}$.

2.2. There exists a series of o. d.'s $\alpha=\alpha_{0}, \alpha_{1}, \cdots, \alpha_{n}=\beta$ such that $\alpha_{k}$ is the maximal component of a $\xi_{k}$-section of $\alpha_{k-1}$ in the sense of $<_{\xi_{k}}$ for every $k(k=1,2, \cdots, n)$.

3. Let $\xi$ be an o.d. $\xi ¥ 0$ is called the successor of $\xi$ and sometimes denoted as $\xi^{\prime}$. (It is clearly seen that no o. d. lies between $\xi$ and $\xi \# o$ for $<_{\eta}$ where $\eta$ is an o.d. or $\infty$ ). An o.d. $\xi$ is called a $l . o . d$. (limit ordinal diagram constructed from $I$ ), if every component of $\xi$ is different from $o$.

4. Let $\alpha$ be an o. d. and $\xi$ be an o. d. accessible for $<_{o}$. We define ' $\alpha$ is a $\xi$-fan' and ' $\alpha$ is $\xi$-accessible' by transfinite induction on $\xi$ for $<_{o}$ as follows:

4.1. An o.d., every value of which is accessible for $<_{o}$, is an $o$-fan.

4.2. $\alpha$ is $\xi$-accessible, if and only if $\alpha$ is a $\xi$-fan and accessible for $<_{o}$ in the system of $\xi$-fans.

4.3. $\alpha$ is $\xi \# o$-fan, if and only if $\alpha$ is a $\xi$-fan and every $\xi$-section of $\alpha$ is $\xi$-accessible.

4.4. Let $\xi$ be a 1.o.d. $\alpha$ is a $\xi$-fan, if and only if $\alpha$ is an $\eta$-fan for every $\eta$ satisfying $\eta<_{o} \xi$.

Let $\alpha$ be an o.d. $\alpha$ is called an $\infty$-fan, if $\alpha$ is a $\xi$-fan for every o.d. $\xi$ accessible for $<_{o}$, and is called to be $\infty$-accessible, if $\alpha$ is an $\infty$-fan and accessible for $<_{\infty}$ in the system of $\infty$-fans.

The following propositions are easily proved.

Proposition 1. Let $\alpha$ and $\xi$ be o.d.'s. If every o.d. less than $\alpha$ in the sense of $<_{\xi}$ is accessible for $<_{\xi}$, then $\alpha$ is accessible for $<_{\xi}$.

Proposition 2. Let $\alpha$ and $\xi$ be o.d.'s. If $\alpha$ is accessible for $<_{\xi}$, then every o.d. less than $\alpha$ in the sense of $<_{\xi}$ is accessible for $<_{\xi}$.

PROPOSITION 3. Let $\alpha_{1}, \cdots, \alpha_{n}$ and $\xi$ be o.d.'s. If $\alpha_{1}, \cdots, \alpha_{n}$ are accessible for $<_{\xi}$, then $\alpha_{1} \# \cdots \# \alpha_{n}$ is accessible for $<_{\xi}$.

These propositions remain correct, if we replace 'o.d. $\xi$ ', 'o.d.'s $\alpha, \alpha_{1}, \cdots, \alpha_{n}$ ' and 'accessible for $<_{\xi}$ ' by ' 0 . d. $\xi$ accessible for $<_{o}$ ', ' $\xi$-fans $\alpha, \alpha_{1}, \cdots, \alpha_{n}$ ' and ' $\xi$-accessible', respectively. We refer to thus replaced propositions as Propo- 
sitions $1^{*}-3^{*}$.

Proposition 4. Let $\xi$ be an o.d. accessible for $<_{0}$. If $\alpha$ is $\xi \#$ \#-accessible, then $\alpha$ is $\xi$-accessible.

Proof. $\alpha$ is a $\xi$-fan by the definition. We may assume that every $\xi^{\prime}$-fan $\beta$ satisfying $\beta<_{\xi^{\prime}} \alpha$ is $\xi$-accessible. We shall prove that every $\xi$-fan $\beta$ such that $\beta<_{\xi} \alpha$ is $\xi^{\prime}$-fan and $\xi$-accessible by induction on the rank of $\beta$. Let $\beta$ be a $\xi$-fan such that $\beta<_{\xi} \alpha$. If $\beta$ has a $\xi$-section $\beta_{0}, \beta_{0}$ is a $\xi$-fan and $\beta_{0}<_{\xi} \alpha$. Then $\beta_{0}$ is $\xi$-accessible by the hypothesis of induction. We see that $\beta$ is a $\xi^{\prime}$-fan, whether $\beta$ has a $\xi$-section or not. Then one of the following conditions holds :

(1) $\beta<_{\xi^{\prime}} \alpha$.

(2) There exists a $\xi$-section $\alpha_{0}$ of $\alpha$ such that $\beta \leqq_{\xi} \alpha_{0}$.

In the former case, $\beta$ is $\xi$-accessible by our assumption. In the latter case, since $\alpha_{0}$ is $\xi$-accessible, $\xi$-accessibility of $\beta$ follows from Proposition $1^{*}$, q.e.d.

PROPOSITION 5. Let $\xi$ be a l.o.d. accessible for $<_{o}$, and the following condition (C) be satisfied:

(C) For any $\eta, \zeta$ such that $\eta<_{o} \zeta<_{0} \xi$, every $\zeta$-accessible $\xi$-fan is $\eta$-accessible. Then ' $\alpha$ is $\xi$-accessible' implies ' $\alpha$ is $\eta$-accessible' for every $\eta$ less than $\xi$.

Proof. Let the condition (C) be astisfied and $\alpha$ be $\xi$-accessible. Let $\xi_{0}$ be the successor of the greatest index less than $\xi$. We have only to prove that $\alpha$ is $\eta$-accessible for every $\eta$ such that $\xi_{0} \leqq_{0} \eta \leqq_{0} \xi$. We shall prove this by transfinite induction for $<_{\xi}$ on $\alpha$. We may assume that every $\xi$-fan such that $\beta<_{\xi} \alpha$ is $\zeta$-accessible for every $\zeta$ less than $\xi$. For the proof we define an auxiliary notion ' $\gamma$ is the $n$-th $\eta$-branch of $\beta$ with respect to $\zeta_{0}$ and $\zeta_{1}$ ' recursively as follows:

5.1. If $\zeta_{0} \leqq_{0} \eta<_{o} \zeta_{1}$ and $\gamma \subset_{\eta} \beta, \gamma$ is the 1 st $\eta$-branch of $\beta$ with respect to $\zeta_{0}$ and $\zeta_{1}$.

5.2. Let $\gamma \subset_{\eta} \delta$ and $\delta$ be the $n$-th $\zeta$-branch of $\beta$ with respect to $\zeta_{0}$ and $\zeta_{1}$. If $\zeta_{0} \leqq{ }_{0} \eta<_{0} \zeta$, then $\gamma$ is the $n$-th $\eta$-branch of $\beta$. If $\zeta \leqq{ }_{0} \eta<_{0} \zeta_{1}$ then $\gamma$ is the $n+1$-st $\eta$-branch of $\beta$ with respect to $\zeta_{0}$ and $\zeta_{1}$.

Let $\eta$ satisfy $\xi_{0} \leqq{ }_{o} \eta<_{o} \xi$, and $\beta$ be an $\eta$-fan and $\beta<_{\eta} \alpha$. We shall prove that $\beta$ is a $\xi$-fan and $\zeta$-accessible of every $\zeta$ such that $\xi_{0} \leqq{ }_{0} \zeta<_{0} \xi$ by induction on the number of branches of $\beta$ with respect to $\xi_{0}$ and $\xi$. Let $\beta_{0}$ be an arbitrary $\zeta_{0}$-branch of $\beta\left(\xi_{0} \leqq_{0} \zeta_{0}<_{o} \xi\right)$. Using the hypothesis of induction, we see that $\beta_{0}$ is a $\xi$-fan. $\beta_{0}<_{\xi} \alpha$ holds by means of $\beta<_{\eta} \alpha$. Then $\beta_{0}$ is $\zeta_{0}$-accessible by the hypothesis of transfinite induction for $<_{\xi}$. Thus we may consider $\beta$ as a $\xi$-fan. $\beta<_{\xi} \alpha$ holds by means of $\beta<_{\eta} \alpha$. Then $\beta$ is $\zeta$-accessible for every $\zeta$ less than $\xi$ by the hypothesis of transfinite induction. From this our proposition follows by Proposition 1*.

q. e. d.

By Propositions 4 and 5, we see easily 
Proposition 6. Let $\xi$ be an o.d. accessible for $<_{o}$ and the condition (C) hold. Then for every $\eta$ less than $\xi$, ' $\alpha$ is $\xi$-accessible' implies ' $\alpha$ is $\eta$-accessible'.

Proposition 7. The condition (C) holds for an arbitrary o.d. $\xi$ accessible for $<_{0}$.

Proof. We prove this by transfinite induction on $\xi$. Suppose now the proposition holds for every $\xi_{0}$ less than $\xi$. If $\xi$ is a $l .0$.d., our assertion is clear by the definition of $\xi$-fan. If $\xi=\zeta_{0} \# o$, our assertion holds for $\zeta$ less than $\zeta_{0}$ by the hypothesis of induction and for $\zeta=\zeta_{0}$ by Proposition 6 .

From Propositions 6 and 7 follows

Proposition 8. Let $\xi$ be an o.d. accessible for $<_{o}, \alpha$ be $\xi$-accessible and $\eta<_{0} \xi$. Then $\alpha$ is $\eta$-accessible.

From Proposition 8 follows

Proposition 9. For any o.d.'s $\eta, \zeta$ accessible for $<_{0}$ and $\eta<_{0} \zeta$ every $\zeta$ accessible $\infty$-fan is $\eta$-accessible.

Proposition 10. If $\alpha$ is $\infty$-accessible, then $\alpha$ is $\xi$-accessible for every o. $d$. $\xi$ accessible for $<_{0}$.

Proof. Following the proof of Proposition 5, we can prove this by the help of Proposition 9.

By transfinite induction over $I$, we have

Proposition 11. Every $\infty$-fan is $\infty$-accessible.

From Propositions 10 and 11, we see easily

Proposition 12. Every $\infty$-fan is $\xi$-accessible for every $\xi$ accessible for $<_{o}$.

Proposition 13. Every o-fan is $\xi$-accessible where $\xi$ is an arbitrary o.d. accessible for $<_{0}$ or $\xi$ is $\infty$.

We see easily the following proposition.

Proposition 14. Let $\alpha$ and $\beta$ be c.o.d.'s and $\xi$ an o.d. If $\alpha<_{\xi} \beta$, then $\alpha<_{\infty} \beta$ or there exists $a\left(\xi_{1}, \cdots, \xi_{n}\right)$-section $\beta_{0}$ of $\beta$ such that $\xi \leqq{ }_{o} \xi_{1}$ and $\alpha \leqq \infty \beta_{0}$.

Then we have

Proposition 15. Every value of an o.d. $\alpha$ is less than $\alpha$.

Proposition 16. Let $\alpha$ be an o.d. and not an o-fan. Then there exists an o-fan $\beta$ such that $\beta<_{o} \alpha$ and $\beta$ is not accessible for $<_{0}$.

Proof. We prove this by induction on the rank of $\alpha$. By the hypothesis of the proposition, there exists a value $\alpha_{0}$ of $\alpha$ not accessible for $<_{o}$. We have $\alpha_{0}<_{0} \alpha$ by Proposition 15. If $\alpha_{0}$ is an $o$-fan, we can take $\alpha_{0}$ as $\beta$. If $\alpha_{0}$ is not an $o$-fan, there exists an $o$-fan $\beta$ such that $\beta<_{o} \alpha_{0}$ and $\beta$ is not accessible for $<_{o}$ by the hypothesis of induction. Then $\beta$ has the required property.

Proposition 17. Every o-fan is accessible for $<_{o}$. q.e.d.

Proof. We prove this by transfinite induction for $<_{0}$ on the system of $o$-fans (cf. Proposition 13). Let $\alpha$ be an $o$-fan. We may assume that every 
$o$-fan $\beta$ less than $\alpha$ is accessible for $<_{0}$. Under this hypothesis and Proposition 16, we see easily that, if $\gamma<_{0} \alpha$ then $\gamma$ is an $o$-fan. Then we have the proposition by Proposition 1 .

Proposition 18. Every o.d. is an o-fan.

Proposition 19. Every o.d. is accessible for $<_{o}$.

THEOREM. Every o.d. is accessible for $<_{\xi}$, where $\xi$ is an arbitrary o. $d$. or $\infty$.

Proof. It follows from Propositions 18, 19 and 13.

\section{§3. Relations between $\operatorname{Od}(I, A)$ and $\operatorname{Od}(I)$.}

In this section we shall show that $\operatorname{Od}(I, I)$ is embedded into $\operatorname{Od}(J)$, where $J$ is a union of two sets isomorphic to $I$.

1. Let $I$ be well-ordered, $<$ be the well-ordering of $I$, and the first element of $I$ be denoted by $o$.

We define $\tilde{I}$ to be a set consisting of all the $i$ and $\tilde{i}$ where $i \in I$. $\quad \tilde{<}$ is a well-ordering of $\tilde{I}$, which is defined as follows:

1.1. If $i<j$, then $i \tilde{<} j$.

1.2. If $i \in I$ and $j \in I$, then $i \tilde{<} \tilde{j}$.

1.3. If $i<j$, then $\tilde{i} \tilde{<} \tilde{j}$.

2. In the following some notations (e.g. $\neq, \infty$ ) are used in both $\operatorname{Od}(I, I)$ and $\operatorname{Od}(\tilde{I})$.

Let $\alpha$ be an element of $\operatorname{Od}(I, I) . \quad \alpha^{*}$ is defined recursively as follows :

2.1. If $\alpha \in I$, then $\alpha^{*}$ is $\widetilde{\alpha}$.

2.2. If $\alpha$ is of the form $\left(i, \alpha_{0}, \alpha_{1}\right)$, then $\alpha^{*}$ is $\left(\alpha_{0}^{*},\left(i, \alpha_{1}^{*}\right)\right)$.

2.3. If $\alpha$ is of the form $\alpha_{1} \# \alpha_{2}$, then $\alpha^{*}$ is $\alpha_{1}^{*} \# \alpha_{2}^{*}$.

We see easily the following propositions.

Proposition 1. If $\alpha$ is an element of $\operatorname{Od}(I, I)$, then $\alpha^{*}$ is an element of $\operatorname{Od}(\tilde{I})$.

Proposition 2. Let $\alpha$ and $\beta$ be elements of $\operatorname{Od}(I, I), \alpha^{*}=\beta^{*}$ if and only if $\alpha=\beta$

PROpOSITION 3. If $i$ and $\alpha$ belong to $I$ and $\operatorname{Od}(I, I)$ respectively, then $i<_{\xi} \alpha^{*}$ where $\xi$ is an arbitrary element of $\operatorname{Od}(\tilde{I})$ or $\infty$.

ProOF. We prove this by induction on the rank of $\alpha$. If $\alpha \subseteq I$, then it is clear by 1.2. If $\alpha$ is of the form $\left(j, \alpha_{1}, \alpha_{2}\right)$ then $\alpha^{*}$ is $\left(\alpha_{1}^{*},\left(j, \alpha_{2}^{*}\right)\right)$. By the hypothesis of induction $i<_{0} \alpha_{1}^{*}$, whence follows $i<_{\infty} \alpha^{*}$. Then $i<_{\xi} \alpha^{*}$ for every $\xi \geqq_{0} \alpha_{1}^{*}$. Since $\alpha^{*}$ contains no $\xi$-section such that $\jmath<_{o} \xi<_{o} \alpha_{1}^{*}$, this implies $i<_{\xi} \alpha^{*}$ for $j<_{o} \xi<_{o} \alpha_{1}^{*}$. Since $i<_{j} \alpha_{2}{ }^{*}$ holds by the hypothesis of induction, $i<{ }_{j} \alpha^{*}$ holds. From this we see easily the proposition.

Proposition 4. Let $\alpha$ and $\beta$ be elements of $\operatorname{Od}(I, I)$ and $i \in I . \quad \beta^{*}$ is an $i$-section of $\alpha^{*}$, if and only if $\beta$ is an $i$-section of $\alpha$. 
PROOF. We see easily the proposition by induction on the rank of $\alpha$ and Proposition 3.

Proposition 5. Let $\alpha$ and $\beta$ be elements of $\operatorname{Od}(I, I)$. If $\alpha<_{i} \beta$, then $\alpha^{*}<_{i} \beta^{*}$ where $i \in I$ or $i$ is $\infty$.

Proof. We shall prove this by double induction on the sum of ranks of $\alpha$ and $\beta$ and the number of indices greater than $i$ in $\alpha$ and/or $\beta$.

First we shall prove the case $i=\infty$. We have only to prove $\alpha<_{\infty} \beta$ implies $\alpha^{*}<_{\infty} \beta^{*}$ under the following hypothesis of induction:

(H1) Let $\gamma$ and $\delta$ be any elements of $\operatorname{Od}(I, I)$, and the sum of the ranks of $\gamma, \delta$ be less than the sum of the ranks of $\alpha$ and $\beta$. Then $\gamma<_{j} \delta$ implies $\gamma^{*}<_{j} \delta^{*}$ where $j \in I$ or $j$ is $\infty$.

To show this we separate the cases according to the forms of $\alpha$ and $\beta$. Since other cases are easily treated, we treat here only the case that $\alpha$ and $\beta$ are of the form $\left(i, \alpha_{0}, \alpha_{1}\right)$ and $\left(j, \beta_{0}, \beta_{1}\right)$ respectively. If $\alpha_{0}<_{0} \beta_{0}$, then $\alpha_{0}{ }^{*}<_{0} \beta_{0}{ }^{*}$ by (H1), which implies $\alpha^{*}<_{\infty} \beta^{*}$. If $\alpha_{0}=\beta_{0}$, then we have only to prove $\left(i, \alpha_{1}^{*}\right)<\alpha_{0}^{*}\left(j, \beta_{1}^{*}\right)$ (by Proposition 2), which follows from $\left(i, \alpha_{1}^{*}\right)<_{\infty}\left(j, \beta_{1}{ }^{*}\right)$ (by Proposition 3). $\left(i, \alpha_{1}^{*}\right)<_{\infty}\left(j, \beta_{1}{ }^{*}\right)$ follows from $i<j$, or $i=j$ and $\alpha_{1}{ }^{*}<_{i} \beta_{1}{ }^{*}$ according as $i<j$, or $i=j$ and $\alpha_{1}<_{i} \beta_{1}$.

Then we prove that $\alpha<_{i} \beta$ implies $\alpha^{*}<_{i} \beta^{*}$ for $i \in I$ under (H1) and the following hypothesis of induction:

(H2) $\alpha<_{j} \beta$ implies $\alpha^{*}<_{j} \beta^{*}$ for every $j$ such that the number of indices greater than $j$ in $\alpha$ and/or $\beta$ is less than the number of indices greater than $i$ in $\alpha$ and/or $\beta$.

If there exists an $i$-section $\beta_{0}$ of $\beta$ such that $\alpha \leqq_{i} \beta_{0}$, then $\beta_{0} *$ is an $i$-section of $\beta^{*}$ and $\alpha^{*} \leqq_{i} \beta_{0}^{*}$ by Proposition 4 and (H1). Let $\alpha_{0}<_{i} \beta$ for every $i$-section $\alpha_{0}$ of $\alpha$ and $\alpha<_{j} \beta$ where $j$ is defined as follows: If there exists an index of $\alpha$ and/or $\beta$ greater than $i$, then $j$ is defined to be the minimum of such indices; othewise, $j$ is defined to be $\infty$. Then $\alpha_{0}^{*}<_{i} \beta^{*}$ for every $i$-section $\alpha_{0}^{*}$ of $\alpha^{*}$ and $\alpha^{*}<_{j} \beta^{*}$ by Proposition 4 and (H2). From this follows $\alpha^{*}<_{i} \beta^{*}$ by Proposition 4 .

From these propositions follows

THeOREM 1. $\operatorname{Od}(I, I)$ is embedded into $\operatorname{Od}(\widetilde{I})$.

We define a subsystem $\mathrm{O}(I)$ of $\mathrm{Od}(I)$ recursively as follows:

3.1. If $i \in I$ then $i \in \mathrm{O}(I)$.

3.2. If $i \in I$ and $\alpha \in \mathrm{O}(I)$, then $(i, \alpha) \in \mathrm{O}(I)$.

3.3. If $\alpha \in \mathrm{O}(I)$ and $\beta \in \mathrm{O}(I)$, then $\alpha \# \beta \in \mathrm{O}(I)$.

Then we have

Corollary 1. $\mathrm{O}(I, I)$ is embedded into $\mathrm{O}(\tilde{I})$.

Let $I$ and $A$ be well-ordered. We have the following theorem in the same way as above. 
Theorem 2. If $I$ and $A$ have no element in common, $\operatorname{Od}(I, A)$ is embedded into $\operatorname{Od}(I \cup A)$.

COROllary 2. If $I$ and $A$ have no element in common, $\mathrm{O}(I, A)$ is embedded into $\mathrm{O}(I \cup A)$.

\section{$\S 4$. On a formal theory of $\operatorname{Od}(I, A)$.}

In [5], G. Takeuti proved the consistency of a logical system. We shall consider, the following slight modification of this system: Let $I(a), A(a), a<^{*} b$ and $a \ddot{<} b$ be primitive recursive predicates, and $<^{*}$ and $\ddot{<}$ well-orderings of $I$ and $A$, where $I$ and $A$ are $\{a \mid I(a)\}$ and $\{a \mid A(a)\}$ respectively.

1. Every beginning sequence is of the form $D \rightarrow D$ or of the form $a=b$, $F(a) \rightarrow F(b)$ or a 'mathematische Grundsequènz' in Gentzen [1], or one of the following forms :

$$
\begin{aligned}
& I(a), A_{m}(a, b) \rightarrow G_{m}\left(a, b,\{x, y\}\left(A_{m}(x, y) \wedge x<^{*} a\right)\right) ; \\
& I(a), G_{m}\left(a, b,\{x, y\}\left(A_{m}(x, y) \wedge x<^{*} a\right)\right) \rightarrow A_{m}(a, b) ; \\
& A(a), B_{n}(a, b) \rightarrow H_{n}\left(a, b,\{x, y\}\left(B_{n}(x, y) \wedge x \ddot{<} a\right)\right) ; \\
& A(a), H_{n}\left(a, b,\{x, y\}\left(B_{n}(x, y) \wedge x \ddot{<} a\right)\right) \rightarrow B_{n}(a, b) ;
\end{aligned}
$$

where $m, n=0,1,2, \cdots, A_{0}, A_{1}, \cdots, B_{0}, B_{1}, \cdots$ are symbols for predicate and $G_{m}$ and $H_{n}$ are arbitrary formulas satisfying the following conditions:

(a) $G_{m}(a, b, \alpha)$ and $H_{n}(a, b, \alpha)$ do not contain $A_{m}, A_{m+1}, A_{m+2}, \cdots, B_{0}, B_{1}, B_{2}, \cdots$ and $B_{n}, B_{n+1}, B_{n+2}, \cdots$ respectively.

(b) If $G_{m}(a, b, \alpha)$ or $H_{n}(a, b, \alpha)$ contains a formula of the form $\forall \varphi F(\varphi)$, then $F(\beta)$ contains no bound $f$-variable.

2. The following inference 'induction' is added:

$$
\frac{F(a), \Gamma \rightarrow \Delta, F(a+1)}{F(0), \Gamma \rightarrow \Delta, F(t)}
$$

where $a$ is contained in none of $F(0), \Gamma$ and $\Delta$, and $t$ is an arbitrary term.

3. The inference $\forall$ left on $f$-variable

$$
\frac{F(V), \Gamma \rightarrow \Delta}{\forall \varphi F(\varphi), \Gamma \rightarrow \Delta}
$$

is restricted by the condition that $F(\beta)$ contains no bound $f$-variable.

Then we have the following

THEOREM. This system is consistent.

Proof. Let $J$ be $I \cup A, \prec$ be a well-ordering of $J$ defined as follows :

1. If $i<* j$, then $i<j$.

2. If $i \in I$ and $a \in A$, then $i \prec a$.

3. If $a \ddot{<} b$, then $a<b$.

Then the proof is performed as in [5] considering $J$ as $I$. 
We see easily from the proof of $\S 2$, that the proof for accessibility of $\operatorname{Od}(I, A)$ can be given in a similar way as in $\$ 2$ of [2]. We can develop a formal theory of $\operatorname{Od}(I, A)$ in a subsystem of the above system such that $m=0,1$ and $n=0$. It is noticed that for the consistency-proof for this subsystem, we have only to use $\{\infty\} \cup J_{0} \cup J_{1}$ instead of $J_{\infty}$. We shall not give an exact treatment of the formal theory here, but show how to develop it. First we give all the necessary concepts concerning the construction of $\operatorname{Od}(I, A)$ as the mathematische Grundsequenzen in the same way as in [4], Let $I(a), A(a)$, $a<^{*} b, a \ddot{<} b, O(a),<(i, a, b), \subset(i, a, b)$ and $\leqslant(a, b)$ be the formal counterparts of ' $a \in I$ ', ' $a \in A$ ', ' $a$ is less than $b$ in $I$ ', ' $a$ is less than $b$ in $A$ ', ' $a \in \operatorname{Od}(I, A)$ ', ' $a<_{i} b$ ', ' $a \subset_{i} b$ ' and ' $a<b$ ', respectively. We use further the following abbreviations :

$$
\begin{aligned}
& J^{*}(a) \text { for } \forall \varphi(\forall x(I(x) \wedge \forall y(y<* x \vdash \varphi[y]) \vdash \varphi[x]) \vdash \varphi[a]) ; \\
& D^{*}(a, \alpha) \text { for } \forall x(x<* a \vdash \alpha[x]) \vdash J^{*}(\alpha) ; \\
& \ddot{J}(a) \text { for } \forall \varphi(\forall x(A(x) \wedge \forall y(y \ddot{<} x \vdash \varphi[y]) \vdash \varphi[x]) \vdash \varphi[a]) ; \\
& \ddot{D}(a, \alpha) \text { for } \forall x(x \ddot{<} a \vdash \alpha[x]) \vdash \ddot{J}(a) ; \\
& A(i, \alpha, a) \text { for } \forall \varphi(\forall x(\alpha[x] \wedge \forall y(\alpha[y] \wedge<(i ; y, x) \vdash \varphi[y]) \vdash \varphi[x]) \vdash \varphi[a]) ; \\
& A(i, a) \text { for } A(i,\{x\} O(x), a) \text {; } \\
& \widetilde{O}(a) \text { for } O(a) \wedge \forall x(<(x, a) \vdash A(1, x)) \text {, where } 1 \text { stands for the formal }
\end{aligned}
$$$$
\text { counterpart of the first element of } I \text {; }
$$$$
B(i, a, \alpha) \text { for }
$$$$
I(i) \wedge \tilde{O}(a) \wedge \forall x(x<* i \vdash \alpha[x, a] \wedge \forall y(\subset(x ; y, \alpha) \vdash A(x,\{u\} \alpha[x, u], y))) \text {; }
$$$$
\widetilde{I}(i) \text { for } I(i) \wedge i=0 \text {, where } 0 \text { stands for the formal counterpart of } \infty \text {. }
$$

Then the following sequences are also used as beginning sequences of our system :

1.1. $I(i), C^{*}(i) \rightarrow D^{*}\left(i,\{x\}\left(C^{*}(x) \wedge x<^{*} i\right)\right)$.

1.2. $I(i), D^{*}\left(i,\{x\}\left(C^{*}(x) \wedge x<^{*} i\right)\right) \rightarrow C^{*}(i)$.

1.3. $A(a), \ddot{C}(a) \rightarrow \ddot{D}(a,\{x\}(\ddot{C}(x) \wedge x \ddot{<} a))$.

1.4. $A(a), \ddot{D}(a,\{x\}(\ddot{C}(x) \wedge x \ddot{<} a)) \rightarrow \ddot{C}(a)$.

1.5. $I(i), F(i, a) \rightarrow B(i, a,\{x, y\}(F(x, y) \wedge x<* i))$.

1.6. $I(i), B\left(i, a,\{x, y\}\left(F(x, y) \wedge x<^{*} i\right)\right) \rightarrow F(i, a)$.

We can prove that the sequence $O(a), \widetilde{I}(i) \rightarrow A(i, a)$ is provable in our system. This is done similarly as in [4], using the above proof of accessibility.

Tokyo University of Education 


\section{References}

[1] G. Gentzen, Neue Fassung des Widerspruchsfreiheitsbeweises für die reinen Zahlentheorie, Forschung zur Logik und Grundlegung der exakten Wissenschaften, Neue Folge 4, Leipzig (1938), 19-44.

[2] G. Takeuti, Ordinal diagrams, J. Math. Soc. Japan, 9 (1957), 386-394.

[ 3 ] G. Takeuti, Ordinal diagrams II, J. Math. Soc. Japan, 12 (1960), 385-391.

[4] G. Takeuti, On the Formal Theory of the Ordinal Diagrams, Ann. Japan Assoc. Philos. Sci., 3 (1958), 151-170.

[5] G. Takeuti, On the inductive definition with quantifiers of second order, J. Math. Soc. Japan, 13 (1961), 333-341. 